\title{
QuEF (Quality Evaluation Framework) for Model-Driven Web Methodologies
}

\author{
F.J. Domínguez-Mayo, M.J. Escalona, and M. Mejías \\ Department of Computer Languages and Systems, University of Seville, Spain \\ \{fjdominguez, mjescalona, risoto\}@us.es
}

\begin{abstract}
QuEF (Quality Evaluation Framework) is an environment for the assessment of Model-Driven Web Engineering (MDWE) methodologies. This approach is oriented towards the evaluation, through objectives measures, of the quality of MDWE methodologies in a specific environment. Given the high number of methodologies available and proposed over recent years, it has become necessary to define objective evaluation tools to enable organizations to improve their methodological environment and help designers of web methodologies to design new effective and efficient tools, processes and techniques. Since methodologies are constantly evolving, the need may arise not only to evaluate the quality but also to find out how it can be improved and how the quality improvement process could be optimized in order to reduce costs.
\end{abstract}

Keywords: Model-Driven Web Engineering, Quality, Methodologies, Approaches, Model-Driven Engineering.

\section{Introduction, Problem and Motivation}

In recent years, the growing interest in the internet has led to the generation of a high number of Model-Driven Web Engineering (MDWE) approaches which offer a frame of reference for the Web environment [4]. On the other hand, there are a high number of approaches without standard consensus, [9], 7], [3] a lack in the use of standards, and scarcity of both practical experience and tool support. In the face of this situation, an important need to assess the quality of existing methodologies arises. In this paper, QuEF (Quality Evaluation Framework), an environment for the quality evaluation of Model-Driven Web methodologies, is proposed.

This doctoral consortium paper is organized into the following sections. In Section 2, the aims and objectives of the proposed research is presented. Section 3 presents the research methodology, the work carried out to date, and the tentative plans for future work. In Section 4, the main contributions of the research to Web Engineering are explained.

\section{Aims and Objectives of the Proposed Research}

The main goal of this research is to lay the basis of an environment for the assessment of MDWE methodologies that facilitates a quality assessment and continuous improvement 
of different methodological proposals under some objective criteria in order to improve these methodologies although the framework could be extended to other specific area or domain. Hence, there is a need for the suitable design of MDWE methodologies and effective tools. To this end, our work concentrates on evaluating and comparing existing proposals although the framework could be extended in the future to other areas. On the other hand, the software development process has a direct influence on the quality and cost of software development and, therefore, the use of an MDWE methodology and its influence on the final product quality must be considered.

In this work, an approach, or Methodology, is a Model-Driven proposal for the development of web applications. It may provide a set of guidelines, techniques, processes and/or tools for the structuring of specifications, which are expressed as models. Only web modelling approaches which are based on MDA in the framework are considered. In addition, a framework in this work is a basic conceptual structure composed of a set of elements used to evaluate, in this case, MDWE methodologies although it could be extended to other areas or domains. QuEF is a quality evaluation framework with a set of elements based on existing literature as shown in Figure 1, where four components for the evaluation of the quality of MDWE methodologies can be seen:

- Quality Model component: this includes the basis for the specification of quality requirements with the purpose of evaluating quality. It specifies each element and its purposes.

- Thesaurus \& Glossary component: this includes all the necessary terms to improve the standardization of the access channel and communication between users of different MDWE methodologies.

- Approach Characteristic Template component: this includes the description templates of the input methodology characteristics to be evaluated. It depends on the Quality Model description.

- Quality Evaluation Process component: this includes the definition and specification for carrying out the quality evaluation process.

\section{Research Methodology, Work Carried Out to Date, and Tentative Plans for Future Work}

An action research methodology is going to be followed. Therefore, the first step then is to examine the idea carefully in the light of the means available, this work is divided into several phases in order to develop a first version of the thesis project: Analysis of the State of the Art, Identification, Definition of Concepts, Framework Design and Framework Web 2.0 Tool Support implementation. In next step, more fact-finding about the situation is required. If this first period of planning is successful, two items emerge: namely, "an overall plan" of how to reach the objective and secondly, a decision in regard to the first step of action. Usually this planning has also somewhat modified the original idea. The next step is "composed of a circle of planning, executing, and reconnaissance or fact finding for the purpose of evaluating the results of the second step, and preparing the rational basis for planning the third step, and for perhaps modifying again the overall plan'. This research methodology is oriented to problem-solving in social and organizational settings, in our case are the MDWE research community. 


\subsection{The Quality Model Component}

The Quality Model in QuEF is a set of characteristics, subcharacteristics and metrics, quality factors, quality attributes and the relationships between them, which provides the basis for specifying quality requirements and evaluating quality in a specific domain (in this case, MDWE). In Figure 2, the Quality Model metamodel is shown together with the relations between the different elements in the Quality model, whose elements have already been described and explained in previously published papers [1], 2].

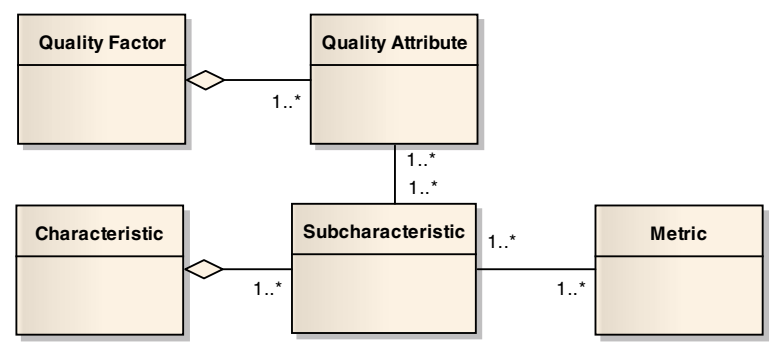

Fig. 1. Quality Model metamodel

In order to define a Quality Model, the metamodel contains association links between the subcharacteristics and the quality attributes. These association links represent the dependencies between subcharacteristics and quality attributes. They show quality attributes which are affected by subcharacteristics or the areas of the methodology that will be significantly affected if the approach is changed. Association links may be based on proven and real-world experience. The impact of each subcharacteristic on quality attributes must be demonstrated and the requirements determined by real case-study applications to a number of real projects. This should be supplemented by reference to published literature. Furthermore, subcharacteristics have to define quantitative or qualitative metrics which may be used to measure each subcharacteristic. Otherwise it would be necessary to define a set of indicators from reference values which may be set to a prescribed state based on the results of measuring or on the occurrence of a specified condition. Hence, a quality factor has various quality attributes and a characteristic has various subcharacteristics, as is shown in Figure 2. A weight is used to define the importance of a metric in the value of a subcharacteristic. Similarly, a weight is also used to define the importance of a quality attribute in the value of a quality factor and the influence importance in association links between subcharacteristics and quality attributes. The tasks for the definition of the Quality Model have already been described in previous papers, and are: The identification quality factors; The identification of quality attributes for each quality factor; The identification of characteristics; The identification of subcharacteristics and metrics for each characteristic; and, The specification of association links between the subcharacteristics and the quality factors. 


\subsection{The Thesaurus and Glossary Component}

An important element for QuEF is the thesaurus component. A thesaurus is a list containing the "terms" used to represent concepts, themes or contents of documents in order to standardize the termonlogy which improves the access channel and communication between users of different MDWE methodologies. We consider it necessary to carry out a standardization of terminology to improve the access channel for communication on MDWE. A set of concepts for MDWE methodologies is currently being described and related.

\subsection{The Approach Characteristic Template Component Based on the Quality Model}

Templates with subcharacteristics and metrics for each characteristic are based on the Quality Model and are used to describe an input methodology. These templates are used as input to QuEF. They are analyzed in the evaluation process and compared with the model quality of the Quality Model component. Templates for MDE, Web Modelling, Tool Support and Maturity have already been developed.

\subsection{The Quality Evaluation Process Component}

The Quality Evaluation Process component contrasts the information from each input approach template with information from the Quality Model. The main purpose of this evaluation is to identify tradeoffs and sensitivity points of the methodology under study. The idea is to determine which aspect needs to be improved on MDWE methodology. A simple evaluation is made with the MS Excel considering weights for metrics, subcharacteristics and quality attributes. However, fuzzy logic is currently being considered in order to improve this evaluation process.

\section{Main Contributions of the Research to Web Engineering}

A quality environment for the assessment of MDWE methodologies is proposed. With regards to the contributions obtained from this research, a framework is required for the improvement of current proposals or even for the development of a new methodology. We consider that the use of QuEF will enhance the quality of products, processes and techniques of approaches. Furthermore, it could be used for optimizing a continuous quality improvement since we can select and improve the minimal number of subcharacteristics which have a majority number of influences in quality attributes using the matrix of influences. Furthermore, in various papers we have evaluated subcharacteristics related with MDE, Maturity and Tool Support of the NDT Methodology, which are required for the measurement of the value of MDWE methodologies in order to be able to assess and improve their Functionality, Usability, Portability and Reliability. Other characteristics such as Web Modelling and Quality Assurance have yet to be described and other quality factors such as Maintainability remain to be described. Therefore the use of QuEF can improve the efficiency and effectiveness of MDWE methodologies since this approach evaluation helps one understand the strengths and weaknesses of a methodology. On the other hand, QuEF could be extended to other areas or domains. Besides, different approaches as NDT, UWE, 
WebML and OOHDM methodology are currently being evaluated. Further characteristics and quality factors have yet to be developed. To this end, Microsoft Excel is currently being employed as a first prototype although a new Web 2.0 tool remains to be developed.

\section{Acknowledgements}

This research has been supported by the project QSimTest (TIN2007-67843-C06_03) and by the RePRIS project of the Ministerio de Educación y Ciencia (TIN200730391-E), Spain.

\section{References}

1. Domínguez-Mayo, F.J., Escalona, M.J., Mejías, M., Torres, A.H.: Towards A Quality Evaluation Framework for Model-Driven Web Engineering Methodologies. In: Proceedings of the 6th International Conference on Web Information Systems and Technologies (WEBIST 2010), vol. 2(1), pp. 191-194 (2010)

2. Domínguez-Mayo, F.J., Escalona, M.J., Mejías, M., Ramos, I., Fernández, L.: A Quality Evaluation Framework for MDWE Methodologies. In: Proceedings of the eighteen International Conference on Software Quality Management (SQM 2010), vol. 1(1), pp. 171-184 (2010)

3. Escalona, M.J., Torres, J., Mejías, M., Gutiérrez, J.J., Villadiego, D.: The treatment of navigation in Web Engineering. In: Advances in Engineering Software, vol. 38, pp. 267282. Elsevier Ld., England (2007)

4. Escalona, M.J., Koch, N.: Requirements Engineering for Web Applications - A comparative study. Journal of Web Engineering 2(3), 193-212 (2004)

5. IEEE Std 610.12-1990. IEEE Standard Glossary of Software Engineering Terminology (1990)

6. ISO- International Organization for Standardization, ISO/IEC 9126-1,

http: / / www.iso.org

7. Kroi $\beta$, C., Koch, N.: UWE Metamodel and Profile, User Guide and Reference. Technical Report 0802. Programming and Software Engineering Unit (PST), Institute for Informatics. Ludwig-Maximilians-Universität München, Germany (2008)

8. Mohagheghi, P., Aagedal, J.: Evaluating Quality in Model-Driven Engineering. In: International Workshop on Modeling in Software Engineering (MISE 2007). IEEE Computer Society, Los Alamitos (2007)

9. Schwinger, W., Retschitzegger, W., Schauerhuber, A., Kappel, G., Wimmer, M., Pröll, B., Cachero Castro, C., Casteleyn, S., De Troyer, O., Fraternali, P., Garrigos, I., Garzotto, F., Ginige, A., Houben, G.-J., Koch, N., Moreno, N., Pastor, O., Paolini, P., Pelechano Ferragud, V., Rossi, G., Schwabe, D., Tisi, M., Vallecillo, A., van der Sluijs, Zhang, G.: A survey on web modeling approaches for ubiquitous web applications. International Journal of web Information Systems 4(3), 234-305 (2008) 